\title{
The Angiogenic Potential of Adipose Mesenchymal Stem Cell-derived Extracellular Vesicles is modulated by Basic Fibroblast Growth Factor
}

Tatiana Lopatina ${ }^{1}$, Aurora Mazzeo ${ }^{1}$, Stefania Bruno ${ }^{2}$, Ciro Tetta ${ }^{3}$, Natalia Kalinina ${ }^{4}$, Renato Romagnoli ${ }^{5}$, Mauro Salizzoni ${ }^{5}$, Massimo Porta ${ }^{1}$ and Giovanni Camussi ${ }^{1 *}$

${ }^{1}$ Department of Medical Sciences, University of Torino, Torino, Italy

${ }^{2}$ Department of Molecular Biotechnology and Health Sciences, University of Torino, Torino, Italy

${ }^{3}$ EMEA LA Medical Board, Fresenius Medical Care, Bad Homburg, Germany

${ }^{4}$ Department of Fundamental Medicine, Lomonosov Moscow State University, Russia

${ }^{5}$ Liver Transplantation Center, University of Torino, Torino, Italy

\section{Abstract}

Objective: The aim of the present study was to investigate the effects of basic fibroblast growth factor (bFGF) on the angiogenic properties of extracellular vesicles (EVs) secreted by adipose-derived mesenchymal stem cells (ASCs) in culture.

Methods: We isolated EVs from ASC (ASC-EVs) cultured with bFGF (FGF-EVs) or without bFGF (b-EVs). We compared the EV angiogenic action on human microvascular endothelial cells (HMEC) in vitro by capillarylike structure formation assay and in vivo by subcutaneous injection of HMEC-containing Matrigel in SCID mice. We analysed micro-RNA composition of EVs by PCR array and we selected two mi-RNAs changed after bFGF stimulation: miR-223 and miR-21. We transfected HMEC with short antisense anti-miR-223 or with mimic miR21 to compensate the action of EVs.

Results: In vitro, the total length and the number of branches of vessel-like structures formed by FGF-EVstimulated HMEC were significantly reduced in respect to b-EV stimulation and the structures were significantly larger than after b-EV stimulation. In vivo, vessels formed by FGF-EV-stimulated HMEC were significantly lower in number in respect to those formed by b-EV-stimulated HMEC, but they were significantly larger in size and contained cells positive for mouse smooth muscle actin. We found that bFGF led to molecular changes in ASCEVs characterized by decreased expression of angiogenic factors. Moreover, bFGF stimulation up-regulated the expression of the anti-angiogenic miR-223 and decreased the level of pro-angiogenic miR-21. The effects of FGFEVs were antagonized by the inhibition of miR-233 and by the miR-21 mimic

Conclusions: The results indicate that culture conditions may modify the pro-angiogenic activity of ASCderived EVs, changing their protein and RNA contents. In particular, bFGF induces production of EVs that stimulate vessel stabilization rather than an increase in vessel number.

Keywords: Adipose mesenchymal stem cells; Extracellular vesicles; bFGF; Angiogenesis

\section{Introduction}

Adipose tissue is constantly growing in the adult organism and is one of the more actively remodelled tissues $[1,2]$. This tissue is well vascularized. Adipose mesenchymal stem cells (ASCs) are located in the vessel walls $[3,4]$ and may regulate angiogenesis and repair processes $[4,5]$. Being easily available by liposuction, ASCs are one of the most promising sources of adult stem cells for cell therapy. However, stem cell employment may be limited by tumorigenesis or maldifferentiation [6]. Recent studies indicated that stem cells enhance angiogenesis and regenerative processes by producing several bioactive molecules (growth factors, cytokines, etc.) [7-10]. The beneficial effect of conditioned medium derived from mesenchymal stem cells supports this paracrine view of stem cells action [11,12]. Previous studies indicated that many of the regenerative functions of adult stem cells are mediated by the release of extracellular vesicles (EVs) [13]. EVs are membrane vesicles in size of nanometers that may act as an intercellular mediator of cell-to-cell communication and, depending of the cell of origin, EVs are able to regulate migration, proliferation, differentiation, angiogenesis, inflammation, and other biological processes [14-20]. All types of EVs carry adhesion molecules and receptors on their surface, which are necessary for their correct contact with the recipient cells and for their entry into them. EVs can fuse with the plasma membrane of target cells releasing their contents inside. EVs transfer not only proteins but also nucleic acids. This leads to activation of defined signalling pathways in the recipient cells. These mechanisms have been described in several regenerative processes, such as angiogenesis [20], liver [21] and kidney repair [22,23].

We recently reported that EVs derived from PDGF stimulated ASCs enhance their angiogenic potential, suggesting that soluble factors acting on ASCs may modulate secretion and function of derived EVs [13]. In the present study we evaluated whether the basic

*Corresponding author: Dr. Giovanni Camussi, Dipartimento di Medicina Interna, Ospedale Maggiore S. Giovanni Battista, Corso Dogliotti 14, 10126 Torino, Italy, Tel: +39-011-6336708; Fax: +39-011-6631184; E-mail: giovanni.camussi@unito.it

Received September 08, 2014; Accepted November 17, 2014; Published November 19, 2014

Citation: Lopatina T, Mazzeo A, Bruno S, Tetta C, Kalinina N, et al. (2014) The Angiogenic Potential of Adipose Mesenchymal Stem Cell-derived Extracellular Vesicles is modulated by Basic Fibroblast Growth Factor. J Stem Cell Res Ther 4: 245. doi:10.4172/2157-7633.1000245

Copyright: (c) 2014 Lopatina T, et al. This is an open-access article distributed under the terms of the Creative Commons Attribution License, which permits unrestricted use, distribution, and reproduction in any medium, provided the original author and source are credited. 
fibroblast growth factor (bFGF) modify the number and function of EVs released from ASCs. bFGF is the first pro-angiogenic molecule identified [24] and it is capable to stimulate survival, proliferation, migration and differentiation of endothelial cells [25]. Moreover, bFGF plays an essential role in expansion, osteogenic and chondrogenic differentiation of mesenchymal stem cells [26]. Based on these considerations, we expected that bFGF would increase the angiogenic characteristics of ASC-derived EVs. Unexpectedly, we found that bFGF instead decreased the angiogenic properties of ASC-EVs by modifying their content of pro- and anti-angiogenic factors.

\section{Methods}

\section{Cell cultivation and EV collection}

ASCs and human microvascular endothelial cells (HMEC) were purchased from Lonza. ASCs were cultured in MSCGM ${ }^{\mathrm{Tx}}$ Mesenchymal Stem Cell Growth Medium (Lonza) containing, 1\% antibioticantimycotic (HyClone) at $37^{\circ} \mathrm{C}$ in $5 \% \mathrm{CO}_{2}$ incubator. Moreover, ASCs were also obtained with informed consent from subcutaneous tissue of non-tumor patients undergoing surgery according to a protocol approved by Ethic committee of the Città della Salute e della Scienza di Torino (Permit number 0012175). ASCs were isolated by enzyme treatment as described previously [9]. For the experiments, ASCs of the 4-6 passages were used. HMEC were cultured in EBM-2 growth medium (Lonza) supplemented with a cocktail of angiogenic factors (SingleQuots; Lonza) according to the instructions of the manufacturer.

For EV collection from ASCs, cells were cultured in DMEM without FBS for 24 hours. Then the medium was replaced with fresh DMEM FBS-free (basal condition, b-EVs) or with DMEM FBS-free containing $20 \mathrm{ng} / \mathrm{ml}$ bFGF (FGF-EVs). Mesenchymal surface markers of ASCs did not change after FBS deprivation, as well as, after stimulation with bFGF (data not shown). Supernatants of ASCs were first centrifuged at $3 \mathrm{k}$ and $10 \mathrm{~kg}$ to remove debris, apoptotic bodies, large vesicles and then submitted to ultracentrifugation for 3 hours at $4^{\circ} \mathrm{C}$ at $100 \mathrm{~kg}$ (Beckman Coulter Optima L-90K ultracentrifuge; Beckman Coulter, Fullerton, CA) as previously described [13]. The pellet was re-suspended in DMEM FBS-free.

Analysis of EV quantity and size distribution was performed using NanoSight LM10 (NanoSight Ltd, Minton Park UK). Using a laser light source the particles in the sample are illuminated, the scattered light is captured by the camera and analysed by Nanoparticle Tracking Analysis (NTA). Using NTA, the particles are automatically tracked and sized based on Brownian motion and the diffusion coefficient (Dt). Results are displayed as a frequency size distribution graph and output to a spreadsheet.

To trace EVs by fluorescent microscopy and to prove that EVs are membrane vesicles and not protein aggregates, they were labelled with the red fluorescent aliphatic chromophore intercalating into lipid bilayers PKH26 dye (Sigma-Aldrich). After labelling, EVs were washed by ultracentrifugation at $100 \mathrm{~kg}$ and re-suspended in DMEM.

\section{HMEC transfection}

For depletion of miR21 and miR223 in HMEC, we transfected the cells using HiPerfect Transfection Reagent (Qiagen) according to the instructions of the manufacturer. Mimic miR-21 (Sigma) and antimiR-223 (Qiagen) were used for transfection. AllStars Negative Control siRNA Alexa 488 (Qiagen, $100 \mathrm{nM}$ ) was used as negative control.

For all in vitro experiments we decided to use concentration of EVs equal to their concentration in ASC conditioned media, when ASCs achieved confluence: this concentration was $10 \times 10^{10}\left( \pm 1 \times 10^{3}\right)$ EVs/ $\mathrm{ml}[13]$.

\section{Secreted protein antibody array}

Purified EVs were lysed in $1 \mathrm{ml}$ of 2x Cell Lysis Buffer (RayBiotech, Inc, GA), and aliquots (1000 mg protein) were added to the "RayBio Biotin Label-based Human Antibody Array I" (RayBiotech, Inc, GA) and processed according to the instructions of the manufacturer. The array provides detection of 507 secreted proteins. The analysis was repeated with three different samples of b-EVs and FGF-EVs. Only proteins detected in all three experiments were mentioned as evidently detected. The biotin-conjugated antibodies on each membrane served as positive controls.

\section{Immunoblotting}

Protein samples were separated by $4 \%$ to $15 \%$ gradient sodium dodecyl sulfate-polyacrylamide gel electrophoresis (SDS PAGE) and subjected to immunoblotting with antibodies to TGFb-1, IGFR1, CD29, Pax6, GAPDH, VEGFA (Santa Cruz). The protein bands were visualized with an enhanced chemiluminescence (ECL) detection kit and ChemiDoc ${ }^{\mathrm{m} n}$ XRS+ System (BioRad). Ten $\mu \mathrm{g} /$ well of cell lysates were loaded.

\section{RNA extraction}

Total RNA was isolated from different EVs using the mirVana RNA isolation kit (Ambion) according to the manufacturer's protocol. Since the intact $18 \mathrm{~S}$ and $28 \mathrm{~S}$ rRNAs were hardly detectable in the EVs, RNA was quantified spectrophotometrically (Nanodrop ND-1000, Wilmington DE).

\section{miRNA profiling by quantitative real-time PCR}

miRNA expression levels were analysed using the Applied Biosystems TaqMan ${ }^{\circledR}$ MicroRNA Assay Human Panel Early Access kit (Applied Biosystems, Foster City, CA) to profile 365 mature miRNAs by qRT-PCR. The kit used gene-specific stem-loop reverse transcription primers and TaqMan probes to detect mature miRNA transcripts in a 2-step real-time reverse-transcription PCR assay. Briefly, single stranded cDNA was generated from total RNA sample $(80 \mathrm{ng})$ by reverse transcription using a mixture of looped primers (Multiplex RT kit, Applied Biosystems) following manufacturer's protocol. The $\mathrm{RT}$ reactions were then diluted and mixed with a Taqman universal master Mix (Applied) in a ratio 1:1, loaded in the TaqMan microfluid card and qRT-PCR experiments were performed. All reactions were performed using an Applied Biosystems 7900HT real-time PCR instrument equipped with a 384 well reaction plate. Raw $\mathrm{Ct}$ values were calculated using the SDS software version 2.3 using automatic baseline and threshold. We have analyzed miR expression in 2 samples of b-EVs and 2 samples of FGF-EVs. Only miRs that were presented in both equal samples were considered as evidently expressed.

We analyzed 2 different samples of b-EVs and 2 of FGF-EVs. All microRNAs that were amplified after 35 cycles of PCR were classified as non-expressed. Only microRNA that were detected or not in both equal samples were taken under consideration. MicroRNAs that were detected in all samples but with difference in threshold cycle $(\mathrm{Ct})$ more than 1.5 were classified as microRNA with changed expression.

qRT-PCR was used to confirm some miRNAs screened by microarray analysis. Briefly, $200 \mathrm{ng}$ of input RNA from all samples were reverse transcribed with the miScript Reverse Transcription Kit 
Citation: Lopatina T, Mazzeo A, Bruno S, Tetta C, Kalinina N, et al. (2014) The Angiogenic Potential of Adipose Mesenchymal Stem Cell-derived Extracellular Vesicles is modulated by Basic Fibroblast Growth Factor. J Stem Cell Res Ther 4: 245. doi:10.4172/2157-7633.1000245

Page 3 of 7

and the cDNA was then used to detect and quantify miRNAs of interest by qRT-PCR using the miScript SYBR Green PCR Kit (all from Qiagen, Valencia, CA, USA). All samples were run in triplicate using $3 \mathrm{ng}$ of cDNA for each reaction as described by the manufacturer's protocol (Qiagen). miRNA specific primers to hsa-miR-221, -99a, -21, -31, and -223 , were used in separate reactions. The snoRNAs, RNU48 and RNU44 were used as positive controls. Negative controls using $10 \mu \mathrm{l}$ of water in place of the RNA were performed alongside each reaction. qRT-PCR was performed using a 48- well StepOne ${ }^{\text {rm }}$ Real Time System (Applied Biosystems). miRNA comparison between the two types of EVs was performed on the relative expression data normalized using the geometric mean value of four of the most stable miRNAs identified in the profiling. Fold change in miRNA expression was calculated based on the normalized mean differences between different types of EVs $\left(2^{-\triangle C t}\right)$. Since in qRT-PCR Ct scores greater than 35 are considered non-specific [27], miRNAs with a raw Ct value greater than 35 were not included in the final data analysis. Relative expression data were then normalized using the mean expression value, calculated on the overall miRNA expression in each array, according to a Ct detection cut-off of 35 PCR cycles as described by Mestdagh et al. [23].

\section{In vitro vessel-like formation assay}

HMECs $\left(30 \times 10^{3}\right.$ cells per well) were seeded onto Matrigel-coated 24-well plates and cultured in DMEM medium without FBS in the presence of $10 \times 10^{10} \mathrm{EVs} / \mathrm{ml}$. As a positive control VEGF $(20 \mathrm{ng} / \mathrm{ml})$ was used. After incubation for $48 \mathrm{~h}$, phase-contrast images (magnification, $\times 100$ ) were recorded and the total length of the network structures was measured using MicroImage analysis system (Casti Imaging, Italy) [28]. The total length per field was calculated in five random fields and expressed as a ratio to the respective control.

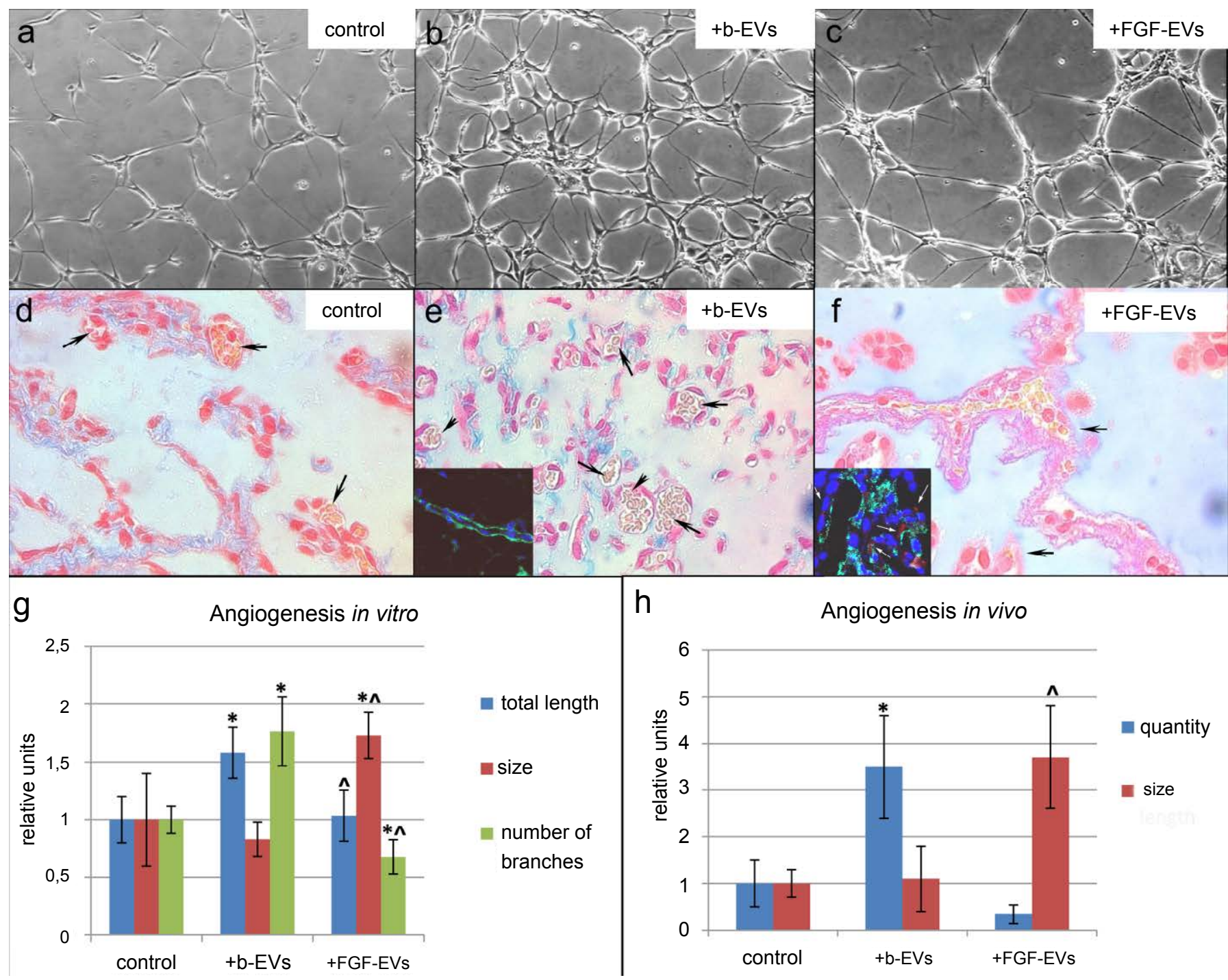

Figure 1: Influence of b-EVs and FGF-EVs on angiogenic properties of HMEC. A, B, C: representative images of HMEC in vitro vessel-like structure formation on Matrigel, (A) without stimulation (control), (B) with b-EV stimulation, or (C) with FGF-EV stimulation; D, E, F : representative in vivo vessel formation by HMEC within Matrigel (sections stained by trichrome method; arrows indicate red blood cell containing vessels); insert shows staining for human CD31 (green) and for mouse smooth muscle actin (red, white arrows); (D) non-stimulated HMEC (control); (E) HMEC stimulated with b-EVs; (F) HMEC stimulated with FGF-EVs. (G) diagram of number and size of in vitro formed vessel-like structures in control and in response to b-EVs or FGF-EVs stimulation (mean \pm SEM, * $-p<0,05$ vs. "control", ^ $p<0,05$ vs. "+b-EVs", $n=15) ;(H)$ diagram of quantity and size of vessels formed within Matrigel by stimulated or non-stimulated HMEC (mean $\pm S E M,{ }^{*}-p<0.05$ vs. "control", $\wedge p<0.05$ vs. "+b-EVs", $n=10)$. 


\section{In vivo angiogenesis assay}

Animal studies were conducted in accordance with the National Institute of Health Guide for the Care and Use of Laboratory Animals. The protocol was approved by the Committee on the Bioethics of the University of Torino (Permit Number: 1.3.10).

Angiogenesis was assayed by measuring the growth of blood vessels from subcutaneous tissue into a solid gel of basement membrane. Firstly, HMEC $\left(1 \times 10^{6}\right.$ cells/injection) were incubated with b-EVs or FGF-EVs $\left(1 \times 10^{10} \mathrm{EVs} / 1 \times 10^{6}\right.$ of HMEC) during 3 hours. Then, male severe combined immunodeficiency (SCID) mice ( 8 week old) were injected subcutaneously with $0.5 \mathrm{ml}$ of ice-cold BD Matrigel Matrix Growth Factor Reduced (BD Biosciences, Franklin Lakes, NJ), which had been mixed with pre-stimulated HMEC. Equivalent quantity of non-stimulated HMEC was used as a negative control. The Matrigel plugs were excised after 10 days and fixed in $4 \%$ paraformaldehyde for 4 h. 5-8 $\mu \mathrm{m}$ thick Matrigel-containing paraffin sections were stained by trichrome stain method [29]. The vessel lumen area (mean size per square millimeter) and quantity of erythrocyte-containing vessels were determined using computerized image analysis software MicroImage analysis system (Casti Imaging).

\section{Statistical analysis}

Data were assessed for normality of distribution using the Kolmogorov-Smirnov test. Statistical analysis was performed using SigmaPlot 11.0 Software. Differences between treatment and control groups were then analysed using Student t-test when the distribution was normal. Data are expressed as mean \pm SEM. We considered differences to be significant when $\mathrm{p}<0.05$.

\section{Results}

bFGF does not affect EVs production by ASCs but influences their angiogenic potential

ASCs in basal conditions (b-EVs) produced about 10,000 $\pm 1,000$ EV per cell. The mean size of b-EVs was $233 \pm 58 \mathrm{~nm}$. These b-EVs did not contain DNA in detectable concentrations and therefore they were not represented by apoptotic bodies. ASC treatment with bFGF did not affect the number $(10,800 \pm 1,300 \mathrm{EV}$ per cell), the size $(245 \pm 79 \mathrm{~nm})$ of FGF-EVs or the presence of apoptotic bodies. Both, PKH26-labelled b-EV and FGF-EVs were internalized by HMEC within 30 minutes (not shown).

b-EVs at concentration of $10 \times 10^{10} \mathrm{EVs} / \mathrm{ml}$ promoted a significant increase of length of vessel-like structures and of the branch number in respect to control HMEC in a manner comparable with VEGF (20 ng/ $\mathrm{ml}$ ) (Figures 1A and 1B). The total length and the number of branches of vessel-like structures formed after stimulation of HMEC with FGFEVs were significantly reduced in respect to b-EV stimulation and did not significantly differed from those of non-stimulated control HMEC (Figure 1C). However, FGF-EVs induced vessel-like structures significantly larger than those of b-EVs (Figures 1C-1G).

HMEC treated b-EVs showed an enhanced ability to induce angiogenesis in vivo in respect to non-stimulated HMEC (Figures 1D1F). New formed vessels contained red blood cells and were positive for human CD31 but not for mouse smooth muscle actin. Vessels formed by HMEC after FGF-EV stimulation were significantly lower in number in respect to those formed by b-EV stimulated HMEC, but they were significantly larger in size and contained cells positive for mouse smooth muscle actin (Figure 1F-1H).

\section{bFGF affects protein and miRNA contents of ASC-derived EVs}

Protein and miRNA content of FGF-EVs and b-EVs was compared. bFGF treatment caused down-regulation of more than $50 \%$ of analyzed proteins. In particular, FGF-EVs lost several angiogenic proteins, including APRIL, artemin, MFG-E8, MMP-20 and angiopoietin-like factor as well as antiangiogenic proteins including trombospondin, angiostatin and endostatin. At the same time 6 new angiogenesismodulatory proteins were found in FGF-EVs, including TRANCE and MMP-7.

Moreover, we compared the miRNA content of b-EVs and FGFEVs using microRNA assay profiling 365 mature miRNAs. b-EVs contained 160 miRNAs, including known pro-angio-miRs, such as miR-126, miR-296, miR-130a, miR-17, miR-21 and miR-210 (Table 1). ASC treatment with bFGF caused the down-regulation of the onethird of microRNAs detected, including 10 microRNAs, which were completely shut-down. The analysis revealed that in FGF-EVs the expression of target miRs for TRANCE (miR-125-3p, miR-194, miR-

\begin{tabular}{|c|c|c|}
\hline $\begin{array}{l}\text { Expressed } \\
\text { only in b-EVs }\end{array}$ & $\begin{array}{c}\text { Expressed in b-EVs two } \\
\text { times more than in FGF- } \\
\text { EVs }\end{array}$ & $\begin{array}{c}\text { Expressed } \\
\text { only in FGF-EVs }\end{array}$ \\
\hline hsa-miR-148a & hsa-let-7a & hsa-let-7c \\
\hline hsa-miR-200c & hsa-let-7b & hsa-miR-130b \\
\hline hsa-miR-222 & hsa-let-7d & hsa-miR-133a \\
\hline hsa-miR-23a & hsa-let-7e & hsa-miR-184 \\
\hline hsa-miR-302c & hsa-let-7g & hsa-miR-193a-3p \\
\hline hsa-miR-329 & hsa-miR-100 & hsa-miR-199b \\
\hline hsa-miR-34c & hsa-miR-101 & hsa-miR-223 \\
\hline hsa-miR-362-3p & hsa-miR-103 & hsa-miR-26b \\
\hline hsa-miR-542-3p & hsa-miR-106a & hsa-miR-27a \\
\hline \multirow[t]{27}{*}{ hsa-miR-652 } & hsa-miR-10a & hsa-miR-28-3p \\
\hline & hsa-miR-10b & hsa-miR-340 \\
\hline & hsa-miR-125a-3p & hsa-miR-381 \\
\hline & hsa-miR-125b & hsa-miR-483-5p \\
\hline & hsa-miR-127 & hsa-miR-539 \\
\hline & hsa-miR-130a & hsa-miR-542-5p \\
\hline & hsa-miR-138 & hsa-miR-545 \\
\hline & hsa-miR-143 & hsa-miR-579 \\
\hline & hsa-miR-15b & hsa-miR-654-3p \\
\hline & hsa-miR-17 & hsa-miR-885-5p \\
\hline & hsa-miR-181a & \\
\hline & hsa-miR-185 & \\
\hline & hsa-miR-192 & \\
\hline & hsa-miR-193a-5p & \\
\hline & hsa-miR-194 & \\
\hline & hsa-miR-195 & \\
\hline & hsa-miR-199a & \\
\hline & hsa-miR-199a-3p & \\
\hline & hsa-miR-20a & \\
\hline & hsa-miR-20b & \\
\hline & hsa-miR-21 & \\
\hline & hsa-miR-210 & \\
\hline & hsa-miR-22 & \\
\hline & hsa-miR-221 & \\
\hline & hsa-miR-224 & \\
\hline & hsa-miR-26a & \\
\hline & hsa-miR-27b & \\
\hline
\end{tabular}

Table 1: MicroRNA content in b-EVs and FGF-EVs. 
Citation: Lopatina T, Mazzeo A, Bruno S, Tetta C, Kalinina N, et al. (2014) The Angiogenic Potential of Adipose Mesenchymal Stem Cell-derived Extracellular Vesicles is modulated by Basic Fibroblast Growth Factor. J Stem Cell Res Ther 4: 245. doi:10.4172/2157-7633.1000245

Page 5 of 7

21, miR-224) and MMP-7 (miR-210) were down-regulated compared with b-EVs.

\section{FGF-EVs modulate HMEC angiogenic properties via miRNAs}

To investigate the relevance of miRNAs in the ability of FGF-EVs to modulate HMEC angiogenic properties we focused on miR-21, a known angiomiR, and on miR-223, a known anti-angiomiR. ASC treatment with bFGF caused down-regulation of miR-21 more than 2 folds and up-regulation of miR-223 up to 2.5 folds in EVs (Figure $2 \mathrm{~A}$ ). In contrast to EVs, the expression of miR-21 and miR-223 in ASCs only showed a tendency to change after bFGF treatment without reaching statistical significance (Figure 2B). The treatment of HMEC with b-EVs caused the elevation of miR-21 expression, whereas FGF-EVs enhanced
miR-223 expression up to 3.5 folds (Figure 2C). These data indicate that ASCs-derived EVs modulate miRNA expression within HMEC.

To confirm the role of these two miRNAs in the ability of FGF-EVs to modulate HMEC angiogenic properties, we transfected HMEC with either mimic miR-21 or antimiR-223 or scramble RNA and $24 \mathrm{hrs}$ after transfection the in vitro angiogenesis test was performed. Transfection of HMEC with mimic mi-R21 enhanced their angiogenic activity in spite of stimulation with FGF-EVs. Inhibition of miR-223 expression in HMEC restored their angiogenic potential even after FGF-EV addition (Figure $3 \mathrm{~A}$ ).

Treatment with FGF-EVs changed the expression of target proteins for miR-21 and miR-223 in HMEC. For example, predicted targets of a

miR21 and miR223 expression in b-EVs and FGF-EVs

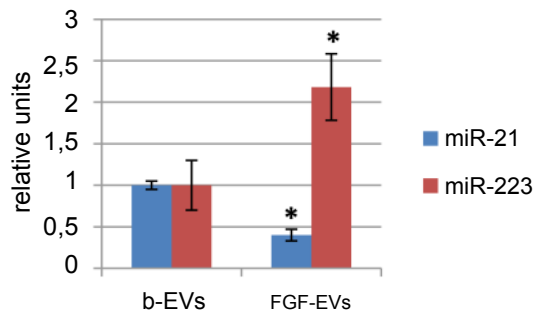

b

miR21 and miR223 expression

in ASC after FGF stimulation

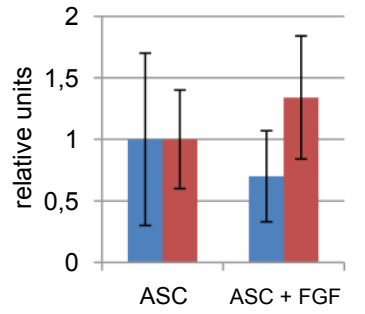

C

miR21 and miR223 expression in HMEC after b-EV or FGF-EV addition

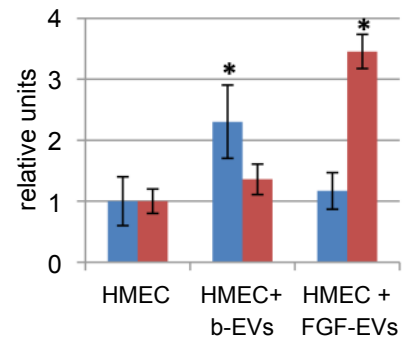

miR-21

miR-223

Figure 2: Expression of miR-21 and miR-223 in EVs, ASCs and HMEC evaluated by Real-time PCR. (a) expression of miR-21 or miR-223 in b-EVs or FGF-EVs, (mean \pm SEM, * $-p<0.05$ vs. "b-EVs", $n=10$ ); (b) expression of miR-21 or miR-223 in ASCs before or after bFGF stimulation; (c) expression of miR-21 or miR-223 in HMEC after EV addition (mean \pm SEM, * $-p<0.05$ vs. "HMEC", $n=10$ ).

A

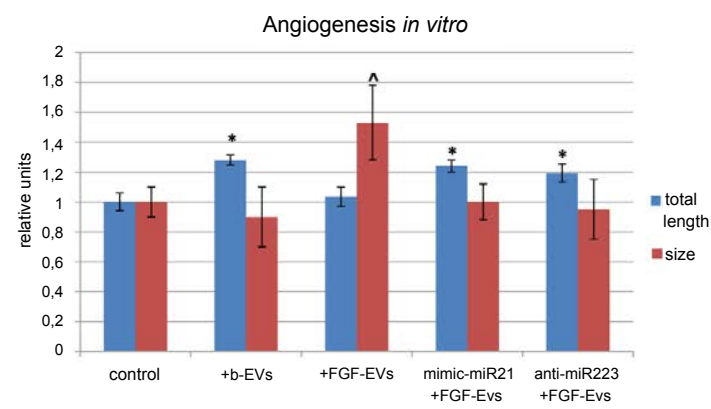

$\mathrm{B}$

C
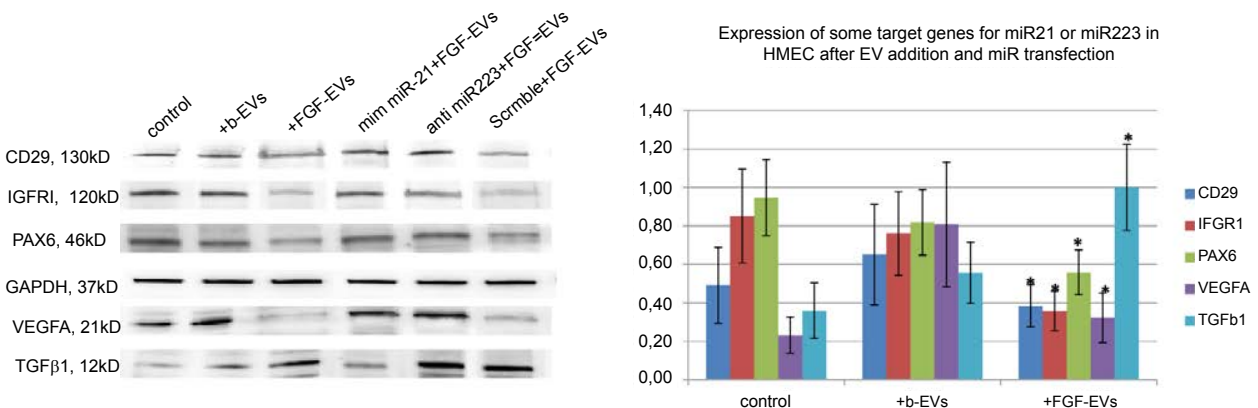

Figure 3: Effect of HMEC transfection with mimic miR-21 or with anti-miR-223. (a) Diagram of vessel-like structures formed in vitro by non-stimulated HMEC (control), by HMEC stimulated with b-EVs or FGF-EVs, or by transfected HMEC stimulated with FGF-EVs. Total length (blue column) and size (red column) of vessel-like structures was measured, (mean \pm SEM, * - p<0,05 vs. "control total length", ${ }^{-}$- $p<0,05$ vs. "control size", $n=15$ ); (b) representative Western blot of proteins, that are targets of miR-21 or miR223; (c) expression of miR-223 or miR-21 target proteins after EV addition (data are represented as densitometric evaluation of band mean intensity \pm SEM, * $-p<0,05$ vs. "+b-EVs", n=5). 
miR-223, CD29, IGFR1 and PAX6 were down-regulated and predicted target of miR-21, TGFb1 was up-regulated in HMEC stimulated with FGF-EVs. Surprisingly, the expression of another predicted target of miR-21, VEGFA was down-regulated in FGF-EV stimulated HMEC (Figures 3B and 3C). These data suggest that EVs could regulate HMEC angiogenic properties through miRNAs.

\section{Discussion}

ASCs are situated in vessel wall and play a key role in the regulation of angiogenesis. It has been demonstrated that these cells may either enhance vessel growth or inhibit vessel formation and stabilize existing vessels. Previous study demonstrated that EVs released by ASCs in basal condition or after stimulation with PDGF possessed proangiogenic activity [13]. In the present study we found that bFGF may negatively modulate the pro-angiogenic activity of ASC-released EVs. This observation further supports the role of soluble factors present in microenvironment in the regulation of content and biological activities of EVs. During tissue injury or remodelling, bFGF plays a critical role. On one side, bFGF directly stimulates endothelial cells to form new vessels. On the other side, bFGF induces differentiation of mesenchymal stem cells (adipogenic, chondrogenic, osteogenic) $[30,31]$. Moreover, bFGF is a factor necessary for mesenchymal stem cell expansion and viability [31,32]. In conditions of wound healing or tissue repair, bFGF plays a complex action that balances angiogenesis with vessel stabilization and maturation. Here we found that addition of bFGF in culture stimulates ASCs to secrete EVs with a reduced angiogenic potential that may favour stabilisation of vessel growth. These functional changes were paralleled by an altered protein and miRNA composition of EVs. In particular we found a down-regulation of the pro-angiogenic miR-21 and down-regulation of anti-angiogenic miR-223. The role of miR21 in angiogenesis is not univocal and depends from target cells and cell environment. In fact, miR21 has been shown to stimulate [33] or suppress [34] angiogenesis through different pathways. MiR-223 is an anti-angiomiR. Overexpression of pre-miR-223 was shown to inhibit endothelial cell migration, tube formation on Matrigel and to prevent sprouting in a modified spheroid assay [35]. MiR-223 has been shown to target beta- 1 integrin and to prevent bFGF-induced migration and sprouting of endothelial cells [35]. We observed that HMEC transfection with mimic miR-21 or antagomiR-223 abrogated the effect of FGF-EVs, suggesting the critical role of these two miRs in the biological activity of ASC-derived EVs.

Inhibitory function of FGF-EVs on angiogenesis may account for the role of ASC in stabilising existing vessels.

\section{Conclusion}

In conclusion, these results indicate that the content and functions of EVs produced from ASCs are modulated by a soluble factor, such as bFGF. Therefore, culture conditions may change the composition and the biological activities of EVs produced by ASCs. This has an implication for eventual production of EVs for therapeutic purposes in case of pathological angiogenesis, such as in tumors, chronic inflammatory disorders, diabetic retinopathy where the pro-angiogenic properties of EVs are unwanted. Understanding of molecular mechanisms of bFGF actions on EV secretion is important for the therapeutic use of this factor.

\section{Acknowledgments}

This work was supported by UNESCO-L'OREAL International Fellowships Program for Young Women in Life Sciences, by a Grant from Fresenius Medical Care, Germany, and by grant from Russian Science Foundation \#14-15-00439.

\section{References}

1. Cao Y (2007) Angiogenesis modulates adipogenesis and obesity. J Clin Invest 117: 2362-2368. [PubMed]

2. Bouloumie A, Lolmede K, Sengenes C, Galitzky J, Lafontan M (2002) Angiogenesis in adipose tissue. Ann Endocrinol (Paris) 63: 91-95. [PubMed]

3. Cai X, Lin Y, Hauschka PV, Grottkau BE (2011) Adipose stem cells originate from perivascular cells. Biol Cell 103: 435-447. [PubMed]

4. Baer PC, Geiger H (2012) Adipose-derived mesenchymal stromal/stem cells tissue localization, characterization, and heterogeneity. Stem Cells Int 2012 812693. [PubMed]

5. Cai L, Johnstone BH, Cook TG, Tan J, Fishbein MC, et al. (2009) IFATS collection: Human adipose tissue-derived stem cells induce angiogenesis and nerve sprouting following myocardial infarction, in conjunction with potent preservation of cardiac function. Stem Cells 27: 230-237. [PubMed]

6. Bergmann A, Steller H (2010) Apoptosis, stem cells, and tissue regeneration Sci Signal 3: re8. [PubMed]

7. Togel F, Westenfelder C (2011) The role of multipotent marrow stromal cells (MSCs) in tissue regeneration. Organogenesis 7: 96-100. [PubMed]

8. Rubina K, Kalinina N, Efimenko A, Lopatina T, Melikhova V, et al. (2009) Adipose stromal cells stimulate angiogenesis via promoting progenitor cell differentiation, secretion of angiogenic factors, and enhancing vessel maturation. Tissue Eng Part A 15: 2039-2950. [PubMed]

9. Lopatina T, Kalinina N, Karagyaur M, Stambolsky D, Rubina K, et al. (2011) Adipose-derived stem cells stimulate regeneration of peripheral nerves: BDNF secreted by these cells promotes nerve healing and axon growth de novo. PLoS One 6: e17899. [PubMed]

10. Bussolati B, Brossa A, Camussi G (2011) Resident stem cells and renal carcinoma. Int J Nephrol: 286985. [PubMed]

11. Angoulvant D, Ivanes F, Ferrera R, Matthews PG, Nataf S, et al. (2011) Mesenchymal stem cell conditioned media attenuates in vitro and ex vivo myocardial reperfusion injury. J Heart Lung Transplant 30: 95-102. [PubMed]

12. Timmers L, Lim SK, Hoefer IE, Arslan F, Lai RC, et al. (2011) Human mesenchymal stem cell-conditioned medium improves cardiac function following myocardial infarction. Stem Cell Res 6: 206-214. [PubMed]

13. Lopatina T, Bruno S, Tetta C, Kalinina N, Porta M, et al. (2014) Plateletderived growth factor regulates the secretion of extracellular vesicles by adipose mesenchymal stem cells and enhances their angiogenic potential. Cell Commun Signal 12: 26. [PubMed]

14. Ratajczak J, Wysoczynski M, Hayek F, Janowska-Wieczorek A, Ratajczak MZ (2006) Membrane-derived microvesicles: important and underappreciated mediators of cell-to-cell communication. Leukemia 20: 1487-1495. [PubMed]

15. Thery C, Ostrowski M, Segura E (2009) Membrane vesicles as conveyors of immune responses. Nat Rev Immunol 9: 581-593. [PubMed]

16. Mathivanan S, Ji H, Simpson RJ (2010) Exosomes: extracellular organelles important in intercellular communication. J Proteomics 73: 1907-1920. [PubMed]

17. Rak J (2010) Microparticles in cancer. Semin Thromb Hemost 36: 888-906. [PubMed]

18. Simons M, Raposo G (2009) Exosomes--vesicular carriers for intercellular communication. Curr Opin Cell Biol 21: 575-581. [PubMed]

19. Cocucci E, Racchetti G, Meldolesi J (2009) Shedding microvesicles: artefacts no more. Trends Cell Biol 19: 43-51. [PubMed]

20. Grange C, Tapparo M, Collino F, Vitillo L, Damasco C, et al. (2011) Microvesicles released from human renal cancer stem cells stimulate angiogenesis and formation of lung premetastatic niche. Cancer Res 71: 5346-5356. [PubMed]

21. Herrera MB, Fonsato V, Gatti S, Deregibus MC, Sordi A, et al. (2010) Human liver stem cell-derived microvesicles accelerate hepatic regeneration in hepatectomized rats. J Cell Mol Med 14: 1605-1618. [PubMed]

22. Camussi G, Cantaluppi V, Deregibus MC, Gatti E, Tetta C (2011) Role of microvesicles in acute kidney injury. Contrib Nephrol 174: 191-199. [PubMed]

23. Gatti S, Bruno S, Deregibus MC, Sordi A, Cantaluppi V, et al. (2011) Microvesicles derived from human adult mesenchymal stem cells protect 
Citation: Lopatina T, Mazzeo A, Bruno S, Tetta C, Kalinina N, et al. (2014) The Angiogenic Potential of Adipose Mesenchymal Stem Cell-derived Extracellular Vesicles is modulated by Basic Fibroblast Growth Factor. J Stem Cell Res Ther 4: 245. doi:10.4172/2157-7633.1000245

against ischaemia-reperfusion-induced acute and chronic kidney injury. Nephrol Dial Transplant 26: 1474-1483. [PubMed]

24. Shing Y, Folkman J, Sullivan R, Butterfield C, Murray J, et al. (1984) Heparin affinity: purification of a tumor-derived capillary endothelial cell growth factor. Science 223: 1296-1299. [PubMed]

25. Cross MJ, Claesson-Welsh L (2001) FGF and VEGF function in angiogenesis: signalling pathways, biological responses and therapeutic inhibition. Trends Pharmacol Sci 22: 201-207. [PubMed]

26. Ng F, Boucher S, Koh S, Sastry KS, Chase L, et al. (2008) PDGF, TGF-beta, and FGF signaling is important for differentiation and growth of mesenchymal stem cells (MSCs): transcriptional profiling can identify markers and signaling pathways important in differentiation of MSCs into adipogenic, chondrogenic, and osteogenic lineages. Blood 112: 295-307. [PubMed]

27. Kedersha N, Anderson $P$ (2007) Mammalian stress granules and processing bodies. Methods Enzymol 431: 61-81. [PubMed]

28. Bussolati B, Deambrosis I, Russo S, Deregibus MC, Camussi G (2003) Altered angiogenesis and survival in human tumor-derived endothelial cells. FASEB J 17: 1159-1161. [PubMed]

29. Goldner J (1938) A modification of the masson trichrome technique for routine laboratory purposes. Am J Pathol 14: 237-243. [PubMed]
30. Kabiri A, Esfandiari E, Hashemibeni B, Kazemi M, Mardani M, et al. (2012) Effects of FGF-2 on human adipose tissue derived adult stem cells morphology and chondrogenesis enhancement in Transwell culture. Biochem Biophys Res Commun 424: 234-238. [PubMed]

31. Chiou M, Xu Y, Longaker MT (2006) Mitogenic and chondrogenic effects of fibroblast growth factor-2 in adipose-derived mesenchymal cells. Biochem Biophys Res Commun 343: 644-652. [PubMed]

32. Itkin T, Kaufmann KB, Gur-Cohen S, Ludin A, Lapidot T (2013) Fibroblast growth factor signaling promotes physiological bone remodeling and stem cell self-renewal. Curr Opin Hematol 20: 237-244. [PubMed]

33. Liu LZ, Li C, Chen Q, Jing Y, Carpenter R, et al. (2011) MiR-21 induced angiogenesis through AKT and ERK activation and HIF-1alpha expression. PLoS One 6: e19139. [PubMed]

34. Sabatel C, Malvaux L, Bovy N, Deroanne C, Lambert V, et al. (2011) MicroRNA-21 exhibits antiangiogenic function by targeting RhoB expression in endothelial cells. PLoS One 6: e16979. [PubMed]

35. Shi L, Fisslthaler B, Zippel N, Fromel T, Hu J, et al. (2013) MicroRNAs-223 Antagonises Angiogenesis by Targeting beta1 Integrin and Preventing Growth Factor Signaling in Endothelial Cells. Circ Res 113: 1320-1330. [PubMed] 